\title{
ON BRENDLE'S ESTIMATE FOR THE INSCRIBED RADIUS UNDER MEAN CURVATURE FLOW
}

\author{
ROBERT HASLHOFER AND BRUCE KLEINER
}

\begin{abstract}
In a recent paper Bre13, Brendle proved that the inscribed radius of closed embedded mean convex hypersurfaces moving by mean curvature flow is at least $\frac{1}{(1+\delta) H}$ at all points with $H \geq C\left(\delta, M_{0}\right)$. In this note, we give a shorter proof of Brendle's estimate, and of a more general result for $\alpha$-Andrews flows, based on our recent estimates from Haslhofer-Kleiner [HK13].
\end{abstract}

\section{INTRODUCTION}

Let $\left\{M_{t} \subset \mathbb{R}^{n+1}\right\}$ be a family of closed embedded mean convex hypersurfaces that evolve by mean curvature flow. In a recent paper [Bre13, Brendle considered the inscribed radius $r_{\text {in }}(p, t)$ and the outer radius $r_{\text {out }}(p, t)$, i.e. the maximal radius of interior respectively exterior balls tangent at $p \in M_{t}$, and proved sharp estimates for them:

Theorem 1.1 (Brendle [Bre13, Thm. 1, Thm. 2]). Let $\left\{M_{t} \subset \mathbb{R}^{n+1}\right\}$ be a smooth family of closed embedded mean convex hypersurfaces which evolve by the mean curvature flow. Then, given any constant $\delta>0$ there exists a constant $C=C\left(\delta, M_{0}\right)<\infty$ such that

$$
r_{\text {in }}(p, t) \geq \frac{1}{(1+\delta) H(p, t)} \quad \text { and } \quad r_{\text {out }}(p, t) \geq \frac{1}{\delta H(p, t)},
$$

whenever $H(p, t) \geq C$.

Brendle's proof is based on some quite sophisticated computations. The purpose of this note is to give a shorter proof of Brendle's estimate.

In fact, we prove a more general theorem (Theorem 1.3), that also applies to certain local and weak solutions. To describe our setting, recall that a mean curvature flow of mean convex hypersurfaces $M_{t}=\partial K_{t}$ satisfies the $\alpha$-Andrews condition [HK13, Def. 1.1], if each boundary point $p \in \partial K_{t}$ admits interior and exterior balls tangent at $p$ of radius at least $\frac{\alpha}{H(p, t)}$; in other words if $\inf \left\{H r_{\text {in }}, H r_{\text {out }}\right\} \geq \alpha$. By the main

Date: January 13, 2018. 
theorem of Andrews And13 (which we extended to weak solutions in [HK13, Thm. 1.5]), the Andrews condition is preserved under the flow, i.e. if $K_{0}$ satisfies the $\alpha$-Andrews condition, then so does $K_{t}$ for all $t \geq 0$. The class of $\alpha$-Andrews flows [HK13, Def. 1.6] is then defined as the smallest class of set flows which contains all compact $\alpha$-Andrews level set flows with smooth initial condition and all smooth $\alpha$-Andrews flows $\left\{K_{t} \subseteq U\right\}_{t \in I}$ in any open set $U \subseteq \mathbb{R}^{n+1}$, and which is closed under the operations of restriction, parabolic rescaling, and passage to Hausdorff limits.

Theorem 1.3. For all $\delta>0, \alpha>0$, there exists $\eta=\eta(\delta, \alpha)<\infty$ with the following property. If $\left\{K_{t}\right\}$ is an $\alpha$-Andrews flow in a parabolic ball $P(p, t, \eta r)$ centered at a boundary point $p \in \partial K_{t}$ with $H(p, t) \leq r^{-1}$, then

$$
r_{\text {in }}(p, t) \geq \frac{r}{1+\delta} \quad \text { and } \quad r_{\text {out }}(p, t) \geq \frac{r}{\delta}
$$

Note that Theorem 1.3 immediately implies Theorem 1.1. More generally, Theorem 1.3 shows that the scale invariant quantities $H r_{\text {in }}$ and $H r_{\text {out }}$ approach the optimal values 1 respectively $\infty$, under the mere assumption that the flow is defined on a parabolic ball that is large enough compared to the curvature scale; the optimal value 1 is attained for the shrinking cylinder $S^{1} \times \mathbb{R}^{n-1}$. Philosophically, the assumption on the domain gives the maximum principle enough room to trigger in, and thus to improve the quantities $H r_{\text {in }}$ and $H r_{\text {out }}$ as much as allowed by the example of the cylinder. In particular, as another interesting consequence of Theorem 1.3. we obtain:

Corollary 1.5. Any ancient $\alpha$-Andrews flow $\left\{K_{t} \subset \mathbb{R}^{n+1}\right\}$ is in fact a 1-Andrews flow.

Corollary 1.5 shows that $\alpha=1$ is the universal noncollapsing constant for ancient mean convex mean curvature flows. It shares some similarities with Perelman's theorem [Per03, 1.5] that any ancient $\kappa$ solution for three-dimensional Ricci flow is either a $\kappa_{0}$-solution or a quotient of the round sphere; our constant is the only sharp one, though.

Our proof of Theorem 1.3 is related to our proof of the convexity estimate in [HK13, Thm. 1.10], see also White [Whi03]. The point is, once the relevant compactness theorem is established (see [HK13, Thm. 1.8] and [HK13, Thm. 1.12] respectively) we can pass to a suitable limit and apply the strong maximum principle. 


\section{THE PROOF}

Proof of Theorem 1.3. As explained in [HK13, Sec. 4] it suffices to establish the estimate for smooth $\alpha$-Andrews flows; the estimate for general $\alpha$-Andrews flows then follows by approximation.

Fix $\alpha<1$. The $\alpha$-Andrews condition implies that the assertion for the inscribed radius holds for $\delta=\frac{1}{\alpha}-1$. Let $\delta_{0} \leq \frac{1}{\alpha}-1$ be the infimum of the $\delta$ 's for which it holds, and suppose $\delta_{0}>0$.

It follows that there is a sequence $\left\{K_{t}^{j}\right\}$ of $\alpha$-Andrews flows, where for all $j, 0 \in \partial K_{0}^{j}, H(0,0) \leq 1$ and $\left\{K_{t}^{j}\right\}$ is defined in $P(0,0, j)$, but $r_{\text {in }}(0,0) \rightarrow \frac{1}{1+\delta_{0}}$ as $j \rightarrow \infty$. By the global convergence theorem [HK13, Thm. 1.12], after passing to a subsequence, $\left\{K_{t}^{j}\right\}$ converges smoothly and globally to an ancient convex $\alpha$-Andrews flow $\left\{K_{t}^{\infty} \subset \mathbb{R}^{n+1}\right\}$. Then for $\left\{K_{t}^{\infty}\right\}$ we have $r_{\text {in }}(0,0)=\frac{1}{1+\delta_{0}}$ and $H(0,0)=1$.

By construction, the quantity $\frac{1}{H r_{\text {in }}}$ attains its maximum over $\partial K_{t}^{\infty}$ for all $t \leq 0$ at $(0,0)$. By Andrews-Langford-McCoy [ALM13] we have the evolution inequality

$$
\partial_{t} \frac{1}{H r_{\text {in }}} \leq \Delta \frac{1}{H r_{\text {in }}}+2\left\langle\nabla \log H, \nabla \frac{1}{H r_{\text {in }}}\right\rangle,
$$

in the viscosity sense. Thus, by the strict maximum principle, $H r_{\text {in }}$ is constant. On the one hand, this constant is equal to $\frac{1}{1+\delta_{0}}$. On the other hand, recalling that the asymptotic shrinkers of $\left\{K_{t}^{\infty}\right\}$ must be cylinders or spheres [HK13, Rem. 1.20], we see that this constant must be at least 1; this gives the desired contradiction and proves the estimate for the inscribed radius.

Finally, the estimate for the outer radius follows from the convexity of ancient $\alpha$-Andrews flows [HK13, Cor. 2.13].

\section{REFERENCES}

[And13] B. Andrews, Noncollapsing in mean-convex mean curvature flow, Geom. Topol. 16(3):1413-1418, 2012

[ALM13] B. Andrews, M. Langford, J. McCoy. Noncollapsing in fully nonlinear curvature flows, Ann. Inst. H. Poincare ANL, 30(1):23-32, 2013.

[Bre13] S. Brendle, A sharp bound for the inscribed radius under mean curvature flow, arXiv:1309.1459, 2013.

[HK13] R. Haslhofer, B. Kleiner, Mean curvature flow of mean convex hypersurfaces, arXiv:1304.0926, 2013.

[Per03] G. Perelman, Ricci flow with surgery on three-manifolds, arXiv:math/0303109, 2013. 
[Whi03] B. White, The nature of singularities in mean curvature flow of mean convex sets, J. Amer. Math. Soc. 16(1):123-138, 2003.

Courant Institute of Mathematical Sciences, New York University, 251 Mercer Street, New York, NY 10012, USA

E-mail: robert.haslhofer@cims.nyu.edu, bkleiner@cims.nyu.edu 